\title{
On the Cartan-Norden theorem for affine Kähler immersions
}

\author{
by MARIA Robaszewska (Kraków)
}

\begin{abstract}
In [O2] the Cartan-Norden theorem for real affine immersions was proved without the non-degeneracy assumption. A similar reasoning applies to the case of affine Kähler immersions with an anti-complex shape operator, which allows us to weaken the assumptions of the theorem given in [NP]. We need only require the immersion to have a non-vanishing type number everywhere on $M$.
\end{abstract}

1. Introduction. Let $M$ be an $n$-dimensional complex manifold with a complex linear torsion-free connection $\nabla$. A holomorphic immersion $f$ : $M \rightarrow \mathbb{C}^{n+1}$ is called an affine holomorphic immersion if there exists a complex transversal bundle $\mathcal{N}$ such that $\nabla$ is induced by its local sections in the following way. For $x \in M$ let $\xi$ be a local section of $\mathcal{N}$ in the neighbourhood $U$ of $x$ (we require all local sections of $\mathcal{N}$ considered to be nowhere vanishing). Let $X, Y$ be vector fields on $U$. Then we have the decomposition

$$
D_{X} f_{*} Y=f_{*} \nabla_{X} Y+h(X, Y) \xi-h(J X, Y) J \xi,
$$

known as the Gauss formula. We denote by $J$ the complex structure on $M$ as well as that on $\mathbb{C}^{n+1}$ and we write $D$ for the standard connection on $\mathbb{C}^{n+1}$. The symmetric bilinear form $h$ is called the affine fundamental form. The Gauss formula may also be written in the complex version

$$
D_{X} f_{*} Y=f_{*} \nabla_{X} Y+h^{c}(X, Y) \xi
$$

with $\mathbb{C}$-bilinear $h^{c}(X, Y)=h(X, Y)-i h(J X, Y)$. The (complex) rank of $h^{c}$ is called the type number of $f$ and denoted by $t f$.

The shape operator $S$ and the transversal forms $\mu, \nu$ are defined on $U$ by the Weingarten formula:

$$
D_{X} \xi=-f_{*} S X+\mu(X) \xi+\nu(X) J \xi .
$$

2000 Mathematics Subject Classification: 53B05, 53C42, 53C55.

Key words and phrases: affine immersion, Kählerian manifold. 
A connection $\nabla$ whose curvature tensor satisfies the condition

$$
R(J X, J Y)=R(X, Y) \quad \text { for all vector fields } X, Y
$$

is called an affine Kähler connection $[\mathrm{NPP}]$ and in this case an affine holomorphic immersion $f:(M, \nabla) \rightarrow\left(\mathbb{C}^{n+1}, D\right)$ is said to be an affine Kähler immersion. The Cartan-Norden theorem deals with Kähler connections, which are known to have the property (1).

We recall the fundamental equations for an affine holomorphic immersion:

$$
\begin{array}{cr}
R(X, Y) Z=h(Y, Z) S X-h(X, Z) S Y-h(J Y, Z) J S X+h(J X, Z) J S Y \\
=h^{c}(Y, Z) S X-h^{c}(X, Z) S Y & (\text { Gauss }), \\
\left(\nabla_{X} h\right)(Y, Z)+\mu(X) h(Y, Z)+\nu(X) h(J Y, Z) & \\
=\left(\nabla_{Y} h\right)(X, Z)+\mu(Y) h(X, Z)+\nu(Y) h(J X, Z) & (\text { Codazzi } I), \\
\left(\nabla_{X} S\right) Y-\mu(X) S Y-\nu(X) J S Y & \\
=\left(\nabla_{Y} S\right) X-\mu(Y) S X-\nu(Y) J S X & (\text { Codazzi II), } \\
h(X, S Y)-h(Y, S X)=2 d \mu(X, Y) & (\text { Ricci }), \\
h(S X, J Y)-h(S Y, J X)=2 d \nu(X, Y) & (\text { Ricci II). }
\end{array}
$$

2. The Cartan-Norden theorem for affine holomorphic immersions with anti-complex shape operator. The theorem to be proved is the following:

ThEOREM. Let $(M, g)$ be a connected, $n$-dimensional, pseudokählerian manifold with the Levi-Civita connection $\nabla$ of $g$. Let $f:(M, \nabla) \rightarrow \mathbb{C}^{n+1}$ be an affine Kähler immersion such that the corresponding shape operator $S$ is anti-complex.

1) If $t f>0$ on $M$, then either $\nabla$ is flat or $R_{x} \neq 0$ for every $x \in M$.

2) If $R_{x} \neq 0$ for every $x \in M$, then there exists a Hermitian product $G$ on $\mathbb{C}^{n+1}$ such that $f:(M, g) \rightarrow\left(\mathbb{C}^{n+1}, G\right)$ is an isometric immersion.

REMARK. The assumption that $S$ is anti-complex is needed only if $t f=1$ everywhere on $M$, because if $\nabla$ is affine Kähler and $t f>1$ at some point of $M$, then the shape operator must be anti-complex [O1].

P r o of. We will first prove the following lemma:

Lemma. Let $W$ be a connected component of the set $\left\{x \in M: R_{x} \neq 0\right\}$. Under the hypotheses of the theorem, there exists a Hermitian product $G$ on $\mathbb{C}^{n+1}$ such that $\left.f\right|_{W}:(W, g) \rightarrow\left(\mathbb{C}^{n+1}, G\right)$ is an isometric immersion. Moreover, if $\xi$ is a transversal vector field inducing the connection $\nabla$ on an open set $U$ and $h, S$ are the affine fundamental form and the shape operator 
associated with $\xi$ respectively, then

$$
h(X, Y)=\frac{1}{G(\xi, \xi)} g(S X, Y)
$$

for all vector fields $X, Y$ on $U \cap W$.

Pr o of. We have divided the proof into a sequence of steps, similar to those in [O2]. In Steps 1-6 we shall prove that there exists a non-vanishing function $\beta$ on $U \cap W$ such that

$$
h(X, Y)=\beta g(S X, Y)
$$

on $U \cap W$, for all vector fields $X, Y$ on $U \cap W$.

STEP 1. If $h_{x} \neq 0$, then $\operatorname{im} R_{x}=\operatorname{im} S_{x}$.

It follows from the Gauss equation that the complex subspace

$$
\operatorname{im} R_{x}=\operatorname{span}\left\{R(X, Y) Z: X, Y, Z \in T_{x} M\right\}
$$

is included in $\operatorname{im} S_{x}$. Let now $X \in T_{x} M$. If $X \in \operatorname{ker} h^{c}$, take $Y, Z$ such that $h^{c}(Y, Z) \neq 0$. Then

$$
S X=\frac{R(X, Y) Z}{h^{c}(Y, Z)} \in \operatorname{im} R_{x} .
$$

If $X \notin$ ker $h^{c}$, take $Y$ such that $h^{c}(X, Y) \neq 0$. Then

$$
R(X, J X) J Y=h^{c}(J X, J Y) S X-h^{c}(X, J Y) S J X=-2 h^{c}(X, Y) S X,
$$

so

$$
S X=\frac{-R(X, J X) J Y}{2 h^{c}(X, Y)} \in \operatorname{im} R_{x} .
$$

SteP 2. If $S_{x} \neq 0$, then $\operatorname{ker} h_{x}=\operatorname{ker} R_{x}$.

We only have to prove that $\operatorname{ker} h_{x} \supset \operatorname{ker} R_{x}$, where

$$
\begin{aligned}
\operatorname{ker} h_{x} & =\left\{X \in T_{x} M: \forall Y \in T_{x} M, h(X, Y)=0\right\}=\operatorname{ker} h_{x}^{c}, \\
\operatorname{ker} R_{x} & =\left\{X \in T_{x} M: \forall Y, Z \in T_{x} M, R(Y, Z) X=0\right\} .
\end{aligned}
$$

Let $Z \in$ ker $R_{x}$ and let $Y \in T_{x} M$.

If $S Y=0$, then for arbitrary $X \in T_{x} M$,

$$
0=R(X, Y) Z=h^{c}(Y, Z) S X .
$$

We can choose $X$ such that $S X \neq 0$, so $h^{c}(Y, Z)=0$.

If $S Y \neq 0$, then the equality

$$
0=R(Y, J Y) J Z=h^{c}(J Y, J Z) S Y-h^{c}(Y, J Z) S J Y=-2 h^{c}(Y, Z) S Y
$$

implies that $h^{c}(Y, Z)=0$.

SteP 3. If $R_{x} \neq 0$, then $\operatorname{ker} h_{x}=\operatorname{ker} S_{x}=\operatorname{ker} R_{x}$.

From the Gauss equation we obtain $S_{x} \neq 0$ and $h_{x} \neq 0$, therefore $\operatorname{ker} h_{x}=\operatorname{ker} R_{x}$ and $\operatorname{im} R_{x}=\operatorname{im} S_{x}$. It remains to prove that $\operatorname{ker} h_{x}=\operatorname{ker} S_{x}$. 
We first show that $\operatorname{dim} \operatorname{ker} S_{x}=\operatorname{dim} \operatorname{ker} h_{x}$.

If $\nabla$ is the Levi-Civita connection for a Kählerian metric $g$, then for all $X, Y, Z, W$ in $T_{x} M$ we have $g(R(X, Y) Z, W)=-g(R(X, Y) W, Z)$, which implies

$$
\left(\operatorname{im} R_{x}\right)^{\perp g}=\operatorname{ker} R_{x}
$$

because of the non-degeneracy of $g$. For the non-degenerate $g$ we have

$$
\begin{aligned}
2 n & =\operatorname{dim}_{\mathbb{R}}\left(\operatorname{im} R_{x}\right)+\operatorname{dim}_{\mathbb{R}}\left(\operatorname{im} R_{x}\right)^{\perp g} \\
& =\operatorname{dim}_{\mathbb{R}}\left(\operatorname{im} R_{x}\right)+\operatorname{dim}_{\mathbb{R}}\left(\operatorname{ker} R_{x}\right) \\
& =\operatorname{dim}_{\mathbb{R}}\left(\operatorname{im} S_{x}\right)+\operatorname{dim}_{\mathbb{R}}\left(\operatorname{ker} h_{x}\right) .
\end{aligned}
$$

On the other hand we have

$$
2 n=\operatorname{dim}_{\mathbb{R}}\left(\operatorname{im} S_{x}\right)+\operatorname{dim}_{\mathbb{R}}\left(\operatorname{ker} S_{x}\right) .
$$

It now remains to prove the inclusion $\operatorname{ker} S_{x} \supset \operatorname{ker} h_{x}$.

Let $X \in \operatorname{ker} h_{x}$. The assertion of Step 2 implies that $X \in \operatorname{ker} R_{x}$. Recall that if $\nabla$ is metrizable and torsion-free, then

$$
\text { ker } R_{x}=\left\{Y \in T_{x} M: \forall X \in T_{x} M, R(X, Y)=0\right\} .
$$

Consequently, for all $Y, Z$ we have $R(X, Y) Z=0$. Using the Gauss equation we obtain

$$
0=h^{c}(Y, Z) S X-h^{c}(X, Z) S Y=h^{c}(Y, Z) S X
$$

for all $Y, Z$. Since $h_{x} \neq 0$, we can find $Y, Z$ such that $h^{c}(Y, Z) \neq 0$, therefore $S X$ must equal 0 .

STEP 4. If $R_{x} \neq 0$, then $S X=0$ implies that $g(S Z, X)=0$ for all $Z \in T_{x} M$.

Let $X \in \operatorname{ker} S_{x}=\left(\operatorname{im} R_{x}\right)^{\perp g}$. Then for all $Y, Z \in T_{x} M$ we have

$$
g(R(J Z, Z) Y, X)=0 \quad \text { and } \quad g(R(J Z, Z) J Y, X)=0 .
$$

Using the Gauss equation we obtain

$$
\begin{aligned}
& h(Z, Y) g(S J Z, X)-h(Z, J Y) g(S Z, X)=0, \\
& h(Z, J Y) g(S J Z, X)+h(Z, Y) g(S Z, X)=0
\end{aligned}
$$

for all $Y, Z \in T_{x} M$. Let $Z \in T_{x} M$. If $Z \in \operatorname{ker} h$, then, by Step $3, Z \in$ ker $S$, so $g(S Z, X)=0$. If $Z \notin$ ker $h$, then there exists $Y \in T_{x} M$ such that $h(Z, Y) \neq 0$. If we now consider (2), we obtain $g(S Z, X)=g(S J Z, X)=0$.

SteP 5. If $R_{x} \neq 0$, then $h(X, Z)=0$ implies $g(S X, Z)=0$.

Let $h(X, Z)=0$. There are three possibilities:

(i) $h(J Z, X) \neq 0$,

(ii) $h(J Z, X)=0$, but $Z \notin \operatorname{ker} h$,

(iii) $Z \in \operatorname{ker} h$. 
If (i) holds, then from the equalities $g(R(X, J X) Z, Z)=0, h(X, Z)=0$ we obtain $h(J X, Z) g(S X, Z)=0$. Hence $g(S X, Z)=0$.

If (ii) holds, then we take $Y$ such that $h(Y, Z) \neq 0$. From

$$
g(R(X, Y) Z, Z)=0 \quad \text { and } \quad g(R(X, J Y) Z, Z)=0
$$

we obtain

$$
\begin{aligned}
& h(Y, Z) g(S X, Z)+h(J Y, Z) g(S J X, Z)=0, \\
& h(J Y, Z) g(S X, Z)-h(Y, Z) g(S J X, Z)=0,
\end{aligned}
$$

which together with $h(Y, Z) \neq 0$ yields $g(S X, Z)=g(S J X, Z)=0$.

If (iii) holds, then, by Step $3, Z \in \operatorname{ker} S$. Hence $g(S X, Z)=0$ by Step 4 .

STEP 6. There exists a non-vanishing function $\beta$ on $U \cap W$ such that

$$
h(X, Y)=\beta g(S X, Y)
$$

on $U \cap W$, for all vector fields $X, Y$ on $U \cap W$.

Let $x \in U \cap W$. The forms $h_{x}(\cdot, \cdot)$ and $g_{x}\left(S_{x}, \cdot\right)$ are bilinear and for all $X, Y \in T_{x} M$ if $h_{x}(X, Y)=0$, then $g_{x}\left(S_{x} X, Y\right)=0$. If that is the case, one can find $\lambda_{x} \in \mathbb{R}$ such that $g_{x}\left(S_{x} \cdot, \cdot\right)=\lambda_{x} h_{x}(\cdot, \cdot)$. Moreover, $\lambda_{x} \neq 0$ because of the non-degeneracy of $g$ and the fact that $S_{x} \neq 0$. For $x \in U \cap W$ we now define $\beta_{x}=1 / \lambda_{x}$. As a function of $x, \beta$ is smooth, because for every $x \in U \cap W$ we can find vector fields $X, Y$ such that $g(S X, Y) \neq 0$ on a neighbourhood $U^{\prime} \subset U \cap W$ of $x$, and then write

$$
\beta=h(X, Y) / g(S X, Y) .
$$

SteP 7. If $h(X, Y)=\beta g(S X, Y)$ on some open subset of $\{x \in M$ : $\left.R_{x} \neq 0\right\}$, then $\beta$ satisfies the following equation:

$$
d \beta+2 \beta \mu=0 .
$$

The proof is the same as in the case of anti-holomorphic transversal bundle in $[\mathrm{NP}]$, the difference is that now we do not have the condition $\nu(X)=\mu(J X)$.

From the first Codazzi equation we have, for all $X, Y, Z$,

$$
\begin{aligned}
0= & \left(\nabla_{X} h\right)(Y, Z)+\mu(X) h(Y, Z)+\nu(X) h(J Y, Z) \\
& -\left(\nabla_{Y} h\right)(X, Z)-\mu(Y) h(X, Z)-\nu(Y) h(J X, Z) \\
= & X(h(Y, Z))-h\left(\nabla_{X} Y, Z\right)-h\left(Y, \nabla_{X} Z\right) \\
& +\mu(X) h(Y, Z)+\nu(X) h(J Y, Z) \\
& -Y(h(X, Z))+h\left(\nabla_{Y} X, Z\right)+h\left(X, \nabla_{Y} Z\right) \\
& -\mu(Y) h(X, Z)-\nu(Y) h(J X, Z) \\
= & X(\beta g(S Y, Z))-\beta g\left(S\left(\nabla_{X} Y\right), Z\right)-\beta g\left(S Y, \nabla_{X} Z\right) \\
& +\mu(X) \beta g(S Y, Z)+\nu(X) \beta g(S J Y, Z)
\end{aligned}
$$




$$
\begin{aligned}
& -Y(\beta g(S X, Z))+\beta g\left(S\left(\nabla_{Y} X\right), Z\right)+\beta g\left(S X, \nabla_{Y} Z\right) \\
& -\mu(Y) \beta g(S X, Z)-\nu(Y) \beta g(S J X, Z) \\
= & X(\beta) g(S Y, Z)+\beta g\left(\left(\nabla_{X} S\right)(Y), Z\right) \\
& -\mu(X) \beta g(S Y, Z)-\nu(X) \beta g(J S Y, Z)+2 \mu(X) \beta g(S Y, Z) \\
& -Y(\beta) g(S X, Z)-\beta g\left(\left(\nabla_{Y} S\right)(X), Z\right) \\
& +\mu(Y) \beta g(S X, Z)+\nu(Y) \beta g(J S X, Z)-2 \mu(Y) \beta g(S X, Z) \\
= & (X(\beta)+2 \mu(X) \beta) g(S Y, Z)-(Y(\beta)+2 \mu(Y) \beta) g(S X, Z) \\
& \left.+\beta g\left(\left(\nabla_{X} S\right)(Y)-\mu(X) S Y-\nu(X) J S Y\right), Z\right) \\
& -\beta g\left(\left(\nabla_{Y} S\right)(X)-\mu(Y) S X-\nu(Y) J S X, Z\right) \\
= & (X(\beta)+2 \mu(X) \beta) g(S Y, Z)-(Y(\beta)+2 \mu(Y) \beta) g(S X, Z),
\end{aligned}
$$

in which the last equality follows from the second Codazzi equation. We have

$$
g((d \beta+2 \beta \mu)(X) S Y-(d \beta+2 \beta \mu)(Y) S X, Z)=0
$$

for all $X, Y, Z$ and from the non-degeneracy of $g$ it follows that

$$
(d \beta+2 \beta \mu)(X) S Y-(d \beta+2 \beta \mu)(Y) S X=0
$$

for all $X, Y$. We claim that

$$
(d \beta+2 \beta \mu)(X)=0
$$

for all $X$.

Let $X \in T_{x} M$. If $S X=0$, then $(d \beta+2 \beta \mu)(X) S Y=0$ for all $Y \in T_{x} M$. Since $S_{x} \neq 0$, we can find $Y$ such that $S Y \neq 0$. Hence (5) holds. If $S X \neq 0$, then we write (4) with $Y=J X$. From the linear independence of $S X$ and $S J X=-J S X$ we conclude that (5) holds.

Using the function $\beta$ we now define a Hermitian product $G_{x}^{\xi}$ on $\mathbb{C}^{n+1}$ :

$$
\begin{aligned}
G_{x}^{\xi}\left(f_{*} X, f_{*} Y\right) & =g(X, Y), \\
G_{x}^{\xi}\left(\xi_{x}, \xi_{x}\right) & =G_{x}^{\xi}\left(J \xi_{x}, J \xi_{x}\right)=1 / \beta_{x} \\
G_{x}^{\xi}\left(f_{*} X, \xi_{x}\right) & =G_{x}^{\xi}\left(f_{*} X, J \xi_{x}\right)=G_{x}^{\xi}\left(\xi_{x}, J \xi_{x}\right)=0
\end{aligned}
$$

for each $x \in U \cap W$.

It is easy to show that if we have two local sections of the transversal bundle $\mathcal{N}, \xi$ and $\widetilde{\xi}=\phi \xi+\psi J \xi$, both defined on some open set $U$, then on $U \cap W$ we have $G^{\xi}=G^{\widetilde{\xi}}$. Indeed, let $x \in U \cap W$. Compare the values of $G_{x}^{\xi}$ and $G_{x}^{\widetilde{\xi}}$, taking all the vectors $f_{*} X$ with $X \in T_{x} M$ and $\widetilde{\xi}_{x}$ as generators of $\mathbb{C}^{n+1}$ :

$$
\begin{aligned}
G_{x}^{\xi}\left(f_{*} X, f_{*} Y\right) & =g(X, Y)=G_{x}^{\widetilde{\xi}}\left(f_{*} X, f_{*} Y\right), \\
G_{x}^{\xi}\left(f_{*} X, \widetilde{\xi}_{x}\right) & =G_{x}^{\xi}\left(f_{*} X, \phi(x) \xi_{x}+\psi(x) J \xi_{x}\right)=0=G_{x}^{\widetilde{\xi}}\left(f_{*} X, \widetilde{\xi}_{x}\right),
\end{aligned}
$$




$$
\begin{gathered}
G_{x}^{\xi}\left(f_{*} X, J \widetilde{\xi}_{x}\right)=-G_{x}^{\xi}\left(f_{*} J X, \widetilde{\xi}_{x}\right)=0=G_{x}^{\tilde{\xi}}\left(f_{*} X, J \widetilde{\xi}_{x}\right), \\
G_{x}^{\xi}\left(\widetilde{\xi}_{x}, \widetilde{\xi}_{x}\right)=G_{x}^{\xi}\left(\phi(x) \xi_{x}+\psi(x) J \xi_{x}, \phi(x) \xi_{x}+\psi(x) J \xi_{x}\right)=\frac{\left(\phi^{2}+\psi^{2}\right)(x)}{\beta_{x}},
\end{gathered}
$$

whereas

$$
G_{x}^{\widetilde{\xi}}\left(\widetilde{\xi}_{x}, \widetilde{\xi}_{x}\right)=1 / \widetilde{\beta}_{x}
$$

It remains to show that

$$
\widetilde{\beta}=\frac{\beta}{\phi^{2}+\psi^{2}} .
$$

Recall that we have

$$
\widetilde{h}(X, Y)=\frac{\phi h(X, Y)-\psi h(J X, Y)}{\phi^{2}+\psi^{2}}, \quad \widetilde{S} X=\phi S X+\psi J S X .
$$

If we put $h(X, Y)=\beta g(S X, Y)$ into the first equation and make use of the anti-complexity of $\mathrm{S}$, we obtain

$$
\widetilde{h}(X, Y)=\frac{\beta}{\phi^{2}+\psi^{2}} g(\phi S X+\psi J S X, Y) .
$$

From the other equation it follows that

$$
\widetilde{h}(X, Y)=\frac{\beta}{\phi^{2}+\psi^{2}} g(\widetilde{S} X, Y) .
$$

Comparing this with the equation $(\widetilde{3})$ defining $\widetilde{\beta}$ we have (6).

We can now define for $x \in W$ a Hermitian product $G_{x}$ on $\mathbb{C}^{n+1}: G_{x}:=$ $G_{x}^{\xi}$, where $\xi$ is a local section of the transversal bundle $\mathcal{N}$ whose domain contains $x$.

Step 8. $G$ is parallel relative to $D$.

Let $X \in T M$. For $Y, Z \in \mathcal{X}(M)$ we have

$$
\begin{aligned}
\left(D_{X} G\right)\left(f_{*} Y, f_{*} Z\right)= & X\left(G\left(f_{*} Y, f_{*} Z\right)\right)-G\left(D_{X} f_{*} Y, f_{*} Z\right)-G\left(f_{*} Y, D_{X} f_{*} Z\right) \\
= & X\left(G\left(f_{*} Y, f_{*} Z\right)\right) \\
& -G\left(f_{*} \nabla_{X} Y+h(X, Y) \xi-h(J X, Y) J \xi, f_{*} Z\right) \\
& -G\left(f_{*} Y, f_{*} \nabla_{X} Z+h(X, Z) \xi-h(J X, Z) J \xi\right) \\
= & X(g(Y, Z))-g\left(\nabla_{X} Y, Z\right)-g\left(Y, \nabla_{X} Z\right) \\
= & \left(\nabla_{X} g\right)(Y, Z)=0, \\
\left(D_{X} G\right)\left(f_{*} Y, \xi\right)= & X\left(G\left(f_{*} Y, \xi\right)\right)-G\left(D_{X} f_{*} Y, \xi\right)-G\left(f_{*} Y, D_{X} \xi\right) \\
= & -G\left(f_{*} \nabla_{X} Y+h(X, Y) \xi-h(J X, Y) J \xi, \xi\right) \\
& -G\left(f_{*} Y,-f_{*} S X+\mu(X) \xi+\nu(X) J \xi\right) \\
= & -h(X, Y) \frac{1}{\beta}+g(Y, S X)=0,
\end{aligned}
$$




$$
\begin{aligned}
\left(D_{X} G\right)(\xi, \xi) & =X(G(\xi, \xi))-2 G\left(D_{X} \xi, \xi\right) \\
& =X(G(\xi, \xi))-2 G\left(-f_{*} S X+\mu(X) \xi+\nu(X) J \xi, \xi\right) \\
& =X\left(\frac{1}{\beta}\right)-2 \mu(X) \frac{1}{\beta} \\
& =-\frac{1}{\beta^{2}}(X(\beta)+2 \beta \mu(X))=0 .
\end{aligned}
$$

Since $D_{X} G$ is hermitian as $G$ is, we conclude that $D_{X} G=0$. As $W$ is assumed to be connected, the map $x \mapsto G_{x}$ is constant on $W$. The proof of the lemma is now complete.

In Steps 9 and 10 we shall prove 1) of the theorem.

STEP 9. If $h_{x} \neq 0$, then $R_{x}=0$ implies $S_{x}=0$.

Let $e_{1}, \ldots, e_{n}$ be a complex basis of $T_{x} M$ such that $h^{c}\left(e_{i}, e_{i}\right)=1$ for $i \in\{1, \ldots, r\}, h^{c}\left(e_{i}, e_{i}\right)=0$ for $i \in\{r+1, \ldots, n\}$ and $h^{c}\left(e_{i}, e_{j}\right)=0$ for $i \neq j$. We have $r>0$ since $h_{x} \neq 0$.

Using the Gauss equation we obtain $0=R\left(e_{j}, e_{1}\right) e_{1}=S e_{j}$ for $j \neq 0$ and $0=R\left(e_{1}, J e_{1}\right) J e_{1}=-2 S e_{1}$.

STEP 10. The connected components of the set $\left\{x \in M: R_{x} \neq 0\right\}$ are closed in $M$.

Let $W$ be a non-empty connected component of $\left\{x \in M: R_{x} \neq 0\right\}$ in $M$. According to the lemma there is a Hermitian product $G$ on $\mathbb{C}^{n+1}$ such that $\left.f\right|_{W}:(W, g) \rightarrow\left(\mathbb{C}^{n+1}, G\right)$ is an isometric immersion. Let $y \in \bar{W}$. Let $U$ be a neighbourhood of $y$ with a local section $\xi$ of the transversal bundle $\mathcal{N}$ and the corresponding $\mathcal{C}^{\infty}$ objects $h, S$ on $U$. On $U \cap W$ we then have a smooth function $\beta: U \cap W \rightarrow \mathbb{R} \backslash\{0\}$ such that $\beta=1 / G(\xi, \xi)$ on $U \cap W$ and $h(X, Y)=\beta g(S X, Y)$ for vector fields $X, Y$ on $U \cap W$.

Define

$$
\alpha: U \ni x \mapsto G\left(\xi_{x}, \xi_{x}\right) \in \mathbb{R}
$$

We claim that

(i) $\alpha \neq 0$ on $\bar{W} \cap U$,

(ii) if $X, Y$ are vector fields on $U$, then

$$
\alpha h(X, Y)=g(S X, Y) \text { on } \bar{W} \cap U .
$$

Let $z \in \bar{W} \cap U$. Let $V$ be an open neighbourhood of $z$ such that $\bar{V} \subset U$; $\bar{W} \cap V \subset \overline{W \cap V}$, since $V$ is open; $\overline{W \cap V} \subset \bar{W} \cap \bar{V} \subset \bar{W} \cap U \subset U$.

For arbitrary vector fields $X, Y$ we define on $U$ the continuous functions $\psi_{1}^{X Y}:=\alpha h(X, Y)-g(S X, Y)$ and $\psi_{2}^{X}:=G\left(f_{*} X, \xi\right)$. Since $\psi_{1}^{X Y}$ and $\psi_{2}^{X}$ vanish on $W \cap V$, we also have $\psi_{1}^{X Y}(\overline{W \cap V})=\{0\}$ and $\psi_{2}^{X}(\overline{W \cap V})=\{0\}$. 
In particular, they vanish at the point $z$. Since $z$ was an arbitrary point from $\bar{W} \cap U$, we have (i) and (ii).

We aim to show that $y \in W$. Since $h_{y} \neq 0$, we can find vectors $X_{y}, Y_{y} \in$ $T_{y} M$ such that $h\left(X_{y}, Y_{y}\right) \neq 0$ and then from the equality

$$
\alpha_{y} h\left(X_{y}, Y_{y}\right)=g\left(S_{y} X_{y}, Y_{y}\right)
$$

we conclude that $S_{y} \neq 0$. Step 9 now implies $R_{y} \neq 0$. If $W_{1}$ denotes the connected component of $\left\{x \in M: R_{x} \neq 0\right\}$ containing $y$, then obviously $W_{1}=W$, so $y \in W$.

From Step 10 and the assumed connectedness of $M$ it follows that if $\nabla$ is not flat, then $R_{x} \neq 0$ for every $x \in M$. The second assertion of the theorem follows immediately from the lemma upon taking $W=M$.

\section{References}

[NPP] K. Nomizu, U. Pinkall and F. Podesta, On the geometry of affine Kähler immersions, Nagoya Math. J. 120 (1990), 205-222.

[NP] K. Nomizu and F. Podesta, On the Cartan-Norden theorem for affine Kähler immersions, Nagoya Math. J. 121 (1991), 127-135.

[O1] B. Opozda, On some properties of the curvature and Ricci tensors in complex affine geometry, Geom. Dedicata 55 (1995), 141-163.

[O2] -, On the Cartan-Norden theorem, Math. Z. 226 (1997), 309-316.

Institute of Mathematics

Jagiellonian University

Reymonta 4

30-059 Kraków, Poland

E-mail: robaszew@im.uj.edu.pl 